\title{
«PASSÉ DÉCOMPOSÉ»? DEUX CRITIQUES DE LA MÉTHODE «GÉNÉTIQUE» DE L'INTERPRÉTATION
}

\author{
Máté PAKSY* \\ Pázmány Péter Universite Catholique, Hungría
}

\begin{abstract}
RESUMEN
Se efectúa una crítica del método de interpretación histórica o genética del Derecho, así como de sus implicaciones para una teoría de la democracia y de la obediencia del orden jurídico dado, con especial detenimiento en las aportaciones de R. Dworkin $y$ de M. Troper.

Palabras clave: Derecho, método de interpretación, interpretación histórica o genética, pensamiento conservador, conservadurismo, Dworkin, Troper.
\end{abstract}

\section{ABSTRACT}

We review the method of historical or genetic interpretation of Law, and its implications for a theory of democracy and the compliance with the provided legal order, with close attention to the contributions by R. Dworkin and M. Troper.

Keywords: Law, Interpretation Method, Historical or Genetic Interpretation, Conservative Line of Thought, Conservatism, Dworkin, Troper.

\section{ZUSAMMENFASSUNG}

Im vorliegenden Aufsatz werden zwei Methodenkritiken der historischen oder «genetischen» Auslegung, sowie ibre Implikationen für eine Demokratietheorie und für den Gehorsams gegenüber der Rechtsordnung unter Berücksichtigung der Beiträge von R. Dworkin und M. Troper ausgeführt.

Schlüsselwörter: Recht, Auslegungsmethode, historische und genetische Auslegung, konservatives Denken, Konservatismus, Dworkin, Troper.

S'il existe un élément constitutif de l'idéologie du conservatisme, c'est certainement la tradition. Cet élément correspond à la dimension temporelle propre à cette idéologie, en l'occurrence le passé ${ }^{1}$.

* ATER, Pázmány Péter Université Catholique, Faculté du droit (Budapest).

${ }^{1}$ Pour ce qui concerne les dimensions temporelles du droit v. en générale François Ost, Le temps du droit, Paris, O. Jacob, 1999, 376 p.; Temps et droit. Le droit a-t-il pour vocation de durer? (Time and law. Is it the nature of law to last?), sous la dir. François OsT et Mark vaN 
Si on cherche un mode d'interprétation juridique, qui représente en particulier le conservatisme juridique, on peut évidemment se référer à l'interprétation «génétique» ou «historique» ${ }^{2}$. C'est parce que, tout d'abord, cette méthode est elle-même baptisée «traditionnelle», étant donné qu'elle a été élaborée en détail par la science juridique allemande sous la base du droit romain. Ensuite, on découvre que le contexte socio-politique de sa genèse est marqué par l'idéologie conservatrice, notamment par l'historicisme (ou le traditionalisme). De l'autre côté du Rhin, le légalisme français, qui tenta de conserver les produits juridiques de la Révolution, se jette aussi dans la même classification idéologique. Le point commun entre les deux est le suivant: sous la bannière de la science juridique positiviste, ils essayaient de forcer le juge à ne consulter que les faits historiques de la genèse de la norme juridique à appliquer. Enfin, focalisant sur ce dernier type de conservativisme juridique, on ajoute que selon cette idéologie, la naissance de la norme juridique n'est pas un phénomène vague comme celui de la Volksgeist en Allemagne, mais procède de la volonté du législateur. La méthode «génétique» suggère que l'interprète est obligé de focaliser sur un fait historique, autrement dit, de chercher l'intention de celui qui produit la norme, en l'occurrence le législateur. Elle est également «positiviste» dans la mesure où elle réduit l'objet de l'interprétation aux actes intentionnels du législateur.

Bien que le temps passe vite, même en théorie du droit, le problème du caractère opératoire et/ou idéologique de la méthode d'interprétation «génétique» et de la nature du positivisme juridique garde un intérêt certain encore aujourd'hui. Il est cependant vrai que prendre position dans le débat sur le caractère conservateur de cette méthode est hautement difficile, car il est incontestable que le conservatisme ancien et moderne, et le positivisme juridique d'hier et d'aujourd'hui ne possèdent que quelques rares points communs.

Quoi qu'il en soi, certains critiquent le conservatisme politique et le positivisme juridique avec la même vigueur, alors que d'autres défendent le positivisme «pur» avec autant de force. Notre étude n'a d'autre ambition que de présenter la critique de la méthode «génétique» conservatrice. Ce qui devrait permettre une meilleure compréhension du positivisme juridi-

Hoecke, Bruxelles, Bruylant, 1998, 469 p.; L'accélération du temps juridique, sous la dir. Philip GÉRARD et al., Bruxelles, Publications des Facultés Universitaires Saint-Louis, 1999, 936 p.

2 Pour une typologie détaillée des méthodes de l'interprétation juridique cf. François Ost et Michel van DE KeRCHOVE, «Interprétation» dans XXV Archive de Philosopbie du Droit, Vocabulaire fondamental du droit (1990), pp. 168-174. 
que (Michel Troper). Afin d'accomplir ce but, nous allons exposer parallèlement quelques remarques antipositivistes (Ronald Dworkin) éclatantes de cette méthode d'interprétation.

1. Pour Ronald Dworkin, professeur américain de grande renommée, ouvertement antipositiviste et anticonservateur, la meilleure philosophie du droit est celle qui «prend les droits au sérieux». En Europe, des théoriciens positivistes peut-être un peu moins connus, mais d'une égale autorité - comme Michel Troper- ${ }^{3}$ ne mettent pas en doute que les droits doivent être pris au «sérieux», mais en les défendant, ils préfèrent ne pas être engagés dans la défense d'une quelconque idéologie. Ainsi, ils prennent d'abord «au sérieux» la science du droit — ce qui n'est pas le cas de Dworkin—, et par-là, les «droits» et les valeurs démocratiques.

Entre ces deux courants, on trouve certes plus de divergences que de convergences. Mais il semble qu'il n'y ait aucun désaccord entre les deux théoriciens à propos d'un problème spécifique de la théorie du droit. En effet, les deux auteurs mettent en cause le caractère opératoire de la méthode «génétique». Ni l'un, ni l'autre n'estime qu'elle est «plausible» ou «vraisemblable». Pour mieux saisir cette critique il convient de préciser la notion de «plausibilité» pour chacun des auteurs. Pour Troper une théorie est «plausible» ou «vraisemblable» seulement si elle correspond à la réalité donc seulement si elle donne une représentation adéquate - susceptible d'êter verifiée ou falsifiée- du phénomène juridique, tandis que pour Dworkin une théorie est «plausible» ou «vraisemblable» seulement si elle est compatible avec une certaine philosophie politique, plus précisément, avec les principes fondamentaux de la Rule of Law.

Alors que le professeur français critique la méthode «génétique» de l'interprétation en ce qu'elle ne correspond pas à la réalité du travail de tous ceux qui possèdent le pouvoir de l'interprète, le philosophe améri-

3 En ce qui concerne la définition du terme «positivisme juridique» cf. en général les chapitres introductifs de son recueil de textes (en collaboration avec Cristoph GRZEGORCZYK et Françoise Michaut) intitulé Le positivisme juridique, Paris-Bruxelles, Libraire Générale de Droit et de Jurisprudence, Story-Scientia, 1993 [La pensée juridique]; «Positivisme juridique» dans André-Jean ARnAud (sous le dir.), Dictionnaire encyclopédique de théorie et de sociologie du droit, Paris, Libraire Générale de Droit et de Jurisprudence, 1993. En particulier «Positivisme juridique» dans PTJE = Pour une théorie juridique de l'Etat, Paris, Presses Universitaires de France, 1994, pp. 27 et s. [Léviathan]; «Science du droit et dogmatique juridique» et «Ross, Kelsen et la validité» toutes les deux dans TDDE = La théorie du droit, le droit, l'Etat, Paris, Presses Universitaires de France, 2001, pp. 3 et s., pp. 19 et s. [Léviathan]; $P h D=$ La philosophie du droit, Paris, Presses Universitaires de France, 2003, pp. 19 et s. [Que sais-je?]; $D C=\{$ avec Francis Hamon $\}$ Droit constitutionnel, Paris, Libraire Générale de Droit et de Jurisprudence, 2001, pp. 27 et s. [Manuels]. 
cain la rejette pour une toute autre raison. Il estime qu'elle ne permet pas au juge de trouver la «seule bonne réponse» de la questio juris, au sens où elle n'est pas un moyen suffisant pour défendre la liberté individuelle.

Outre ce clivage propre aux méthodes d'interprétation, il existe un enjeu idéologique autour de la question particulière de la méthode génétique. De temps à autre, on lui impute de servir un certain conservatisme politique. Certains ont pu soutenir que cette méthode est «positiviste». A cet égard, il est probablement très facile de condamner le positivisme juridique en tant qu'idéologie conservatrice. Cet aspect critique est ouvertement présent dans la doctrine libérale et antipositiviste de Dworkin ${ }^{4}$. En revanche Troper défend le positivisme juridique comme seule théorie compatible avec une théorie de la démocratie et contesterait sa qualification d'idéologie ${ }^{5}$. Si on l'interrogeait sur la liaison entre le positivisme juridique, le conservatisme (ou tout autre idéologie) et la méthode «génétique», il la rejetterait certainement. Sa critique n'est pas substantielle, autrement dit, elle n'indique que les conséquences idéologiques de certains choix des thèses métathéoriques. Le cœur de sa doctrine (sa métathéorie) consiste à énoncer que la science juridique «vraie» n'est rien d'autre qu'un discours neutre sur le droit. Par ailleurs, même du point de vue du discours pragmatique-idéologique, le résultat d'une opération «neutre» du théoricien positiviste demeure cependant très important. Il permet, par la description, de démasquer le véritable mécanisme du pouvoir et permet ainsi de le critiquer.

2. Nous regarderons tout d'abord les aspects idéologiques implicites (paragraphes A et B) et explicites (paragraphe C) d'une méthode d'interprétation et à travers cela certains caractères spécifiques de la doctrine positiviste.

\section{A. La méthode de l'interprétation et la théorie de la démocratie}

Ce rapport nécessaire entre une méthode de l'interprétation et la théorie de la démocratie se fonde sur une logique simple, établie comme suit:

${ }^{4} L E=$ Law's Empire, London, Fontana Press, 1986, pp. 357 et s. [Fontana Masterguides], où Dworkin parle de la distinction entre les juges libéraux et conservateurs. Il attribue aux juges conservateurs deux caractères. D'une part ils préferent une solution qui s'ajuste le plus avec la pratique juridique établie et qui adhère au conservatisme politiqueen proposant, par example, la rétribution comme justification politique de la punition, et d'autre part, ils défendent le liberalisme politique dans la controverse avec le mouvement de Critical Legal Studies qui répresent le scepticisme interne dans la théorie de l'interprétation (ibid., pp. 271 et s.).

5 Cf. les arguments et les definitions dans les ouvrages précités (note 4). 
dans une société démocratique, seule la volonté des individus crée le droit. Les méthodes d'interprétation sont des prescriptions adressées aux juges en ce qui concerne les modes de l'application du droit. Le théoricien est en face d'un choix: soit de respecter la démocratie et sauvegarder la science juridique (descriptive) et dans ce cas là ne pas élaborer les règles d'interprétation, soit de prescrire telle ou telle méthode d'interprétation qui selon lui donne le résultat le plus démocratique possible et d'abandonner alors la science juridique (descriptive). Le risque, dans le premier cas, est la tyrannie des juges qui décident selon leur caprice, dans le deuxième cas, la science juridique pourrait se transforme en une idéologie.

Dworkin pour sa part élabore une doctrine qui est prescriptive selon les deux senses du mot. Composée d'une théorie «substantielle» de la démocratie, elle est accompagnée de la théorie de l'interprétation qui lui est le plus favorable. Par conséquent, Dworkin ne décrit pas comment les juges décident dans les cas difficiles dans une «bonne» démocratie, mais il prescrit bel et bien les moyens d'accès au modèle démocratique. En revanche, il n'y a pas à proprement parler de théorie politique préférée du point de vue de la véritable science (positive). Bien sûr les positivistes comme Kelsen, Ross ou Troper parlent de la démocratie. Ils ne vont pas pour autant plus loin que d'indiquer qu'elle semblerait être la seule méta-éthique —au sens d'un seul concept de philosophie politique- compatible avec le positivisme juridique: «Le positivisme est fondé sur une métaéthique anticognitive et relativiste. Les valeurs n'ayant pas d'existence objective, ne sont pas connaissables, de sorte qu'il n'existe aucun critère permettant d'affirmer la supériorité de certaines d'entre elles [...], si les valeurs ne sont que relatives, alors il n'y a aucune raison pour que le pouvoir appartienne à tel groupe plutôt qu'à un autre. Il faut alors que chacun soit dans la mesure du possible soumis à ses propres valeurs et aux normes qui les fondent, c'est à dire que chacun soit "autonome". Le seul système politique qui ait pour objectif l'autonomie est la démocratie» ${ }^{6}$.

La citation contient une des thèses les plus importantes de ce courant. Elle signifie qu'il est impossible de parler de «raison naturelle» qui pourrait découvrir ou reconstruire les principes «objectifs». Mais selon cette thèse subjectiviste, les fondements des normes juridiques ne sont que des valeurs, la «source» des valeurs est la volonté humaine qui est intentionnelle. Il est tout à fait possible que celui qui a le pouvoir d'interpréter prenne en considération l'intention de l'auteur, mais il s'agit d'un facteur contin-

${ }^{6}$ «Réflexions autour de la question kelsénienne de l'État» dans PTJE, p. 144. 
gent —selon le positivisme juridique— qui n'est pas susceptible d'être justifié ou falsifié empiriquement. Dans l'hypothèse émise par Dworkin, la méthode d'interprétation se doit d'être compatible avec les principes objectifs démocratiques «d'égalité» ou d' «équité». Pour lui, la méthode «génétique» ne donne que partiellement un tel résultat. Troper se positionnant en théoricien n'exprime ni ses préférences à l'égard de la méthode de l'interprétation ni à l'égard du régime politique. Il sauvegarde ainsi l'idée même de la science «neutre» et de la véritable démocratie.

\section{B. Théorie de l'obéissance et la méthode de l'interprétation}

Le deuxième aspect idéologique d'une méthode d'interprétation est la question de l'obligation d'obéissance au droit.

Pour le montrer, prenons comme point de départ un modèle simple de discours juridique: soit un discours pragmatique qui n'est composé que de prescriptions. Ces prescriptions, objet de l'interprétation, déterminent le mode d'interprétation. Elles correspondent à des règles explicitement obligatoires. Si les prescriptions portées sur les prescriptions (donc les règles de l'interprétation) sont positivées - comme par exemple au Canada ou pendant certaines époques en France où la méthode «génétique» était codifiée- elles sont «explicitement» obligatoires. Si, en revanche, elles ne sont pas positivées, elles restent «implicites» et peuvent être utilisées par le juge dans son obligation générale de décider dans chaque cas. Si une recommandation interprétative comme celle de la méthode «génétique» est une norme juridique, celui qui l'interprète se doit d'abord interpréter celle-ci. Le problème de l'obéissance n'est pas éliminé, mais il est donc simplement déplacé. Or le plus souvent, même dans une démocratie, les règles des méthodes de l'interprétation ne sont pas positivées ${ }^{7}$.

Une théorie «plausible» ou «vraisemblable» du droit se doit, nous semble-il, de répondre aussi à la question: «pourquoi les juges doivent obéir aux normes juridiques d'interprétation?». Mais ce n'est pas le cas. Bien au contraire, il faut souligner que seul le positivisme juridique en tant qu'idéologie —ou le «pseudo-positivisme»— ${ }^{8}$ élabore une «théorie de l'obéissan-

7 Pour couper court à la regressison à l'infini, certains trouvent que le pratique contient les ultimes règles de l'interprétation. Cf. Stanley Fish, Doing What Comes Naturally: Change, Rhetoric and the Practice of Theory in Literary and Legal Studies, Durham, N.C. \& Oxford: Clarendon Press \& Duke University Press, 1989, pp. 120-140.

${ }^{8}$ Cf. «Ross, Kelsen et la validité» dans TDDE, pp. 19 et s. 
ce». C'est parce que toutes les théories de l'obéissance sont —au moins partiellement- préscriptives, car l'objet de la recherche dans ce domaine est l'obligation morale d'obéir. Un théorie «positiviste» de l'obéissance devrait déterminer et hiérarchiser les valeurs qui justifient les obligations morales en mettant à l'apex de la hiérarchie des valeurs celles qui servent comme fondement à l'obligation juridique. Les «véritables» positivistes — «prennant au sérieux» les conséquences de la metaéthique anticognitive- transposent cette question au domaine de la philosophe morale (s'ils s'agit d'un discours prescriptif) ou de la sociologie du droit (s'il s'agit d'un discours descriptif). Un tel positionnement épistémologique permet d'établir qu'il n'existe aucune méthode «vraisemblable» — au sens positivistequi indique les motivations ou justifications de l'obéissance des individus (y compris les juges ou les autres détenteurs du pouvoir d'interprétation authentique) $)^{9}$. Ainsi, le positiviste ne risque pas de répondre à la question «pourquoi le juge devrait-il obéir à une norme juridique qui l'oblige à suivre la méthode "génétique" de l'interprétation?» et encore moins à celle de savoir si cette règle n'est pas «valide» ou «positivée». Ceci signifie qu'il n'est pas non plus possible de stipuler une règle catégorique adressée aux juges les obligeant à décider.

En revanche, la théorie du droit se doit aussi de donner une justification ou de créer un fondement théorique pour l'obéissance selon Dworkin. Le théoricien se charge de trouver la meilleure interprétation des pratiques concurrentes qui justifient l'obéissance avec la plus grande force de persuasion possible. C'est nécessaire, parce qu'aux yeux de Dworkin la question primordiale est de justifier la légitimité de la coercition de l'Etat $^{10}$. Dans des cas moralement douteux, produits par des pratiques sociales diverses et parfois même contradictoires, la tâche de trouver «la bonne réponse» obligatoire pour le juge est attribuée au théoricien lorsque la coercition étatique est légitime. La réponse à la questio iuris sera fondée sur la valeur qui soutient la meilleure interprétation de la pratique sociale. La recommandation du théoricien est obligatoire, parce qu'elle seule propose la meilleure justification, indépendamment du fait qu'elle soit positive ou non. Parallèlement, il existe en générale une règle catégorique selon laquelle les juges devraient respecter les critères de la Rule of Law.

\footnotetext{
9 «La liberté d'interprétation du juge constitutionnel» dans ibid., p. 94; «Hans Kelsen et la jurisprudence» dans PTJE, p. 78.

${ }_{10}$ LE, pp. 190 et s.
} 


\section{La critique «explicite» de l'idéologie: «illusionisme» et «originalisme»}

Les deux thèses positivistes qui rejettent l'élaboration d'une théorie «substantielle» de la démocratie et de l'obéissance ne sont que des dérivés de la métathéorie descriptive de la science du droit. Elles s'opposent aussi à la création d'un fondement pour une méthode «génétique» de l'interprétation. Etant donné qu'il s'agit d'une critique dérivée d'un concept de la science juridique, la critique de l'idéologie reste «implicte». Or, Troper la complète avec une critique «explicite» de l'idéologie conservatrice. De façon paradoxale, Dworkin l'antipositiviste reprend à son compte la même critique. Néanmoins, les deux pensées ne se conceptualisent pas dans le même contexte de discours et ne se fondent pas sur les mêmes raisonnements. Tandis que la version française consiste à démasquer une «illusion» commune entre les juges qui ordonnent de ne pas interpréter les normes juridiques, les «inteprétativistes» (antipositivistes-libéraux) américains (comme Dworkin) critiquent les actes de l'interprétation «originaliste» qui se fondent sur l'intention du législateur ${ }^{11}$.

Au fondement de «l'illusionisme» se trouve l'idéologie légaliste conservatrice qui réduit le droit aux lois et qui fait croire au mythe du législateur «parfait». La tâche du juge ne consiste pas à interpréter, mais à appliquer la norme juridique qui possède un sens objectif. Par contre, la science du droit consiste à énumérer les sens possibles d'une norme juridique à appliquer. L'impact est indubitable non seulement sur le Code civil français mais aussi sur la pensée théorique-juridique, qui nous apparaît sous la forme «scientifique» par laquelle l'Ecole exégétique a mis l'interprétation «génétique» au service du pouvoir. Elle suggère que dans un cas «difficile» le juge doive rechercher les intentions du législateur ou avoir recours au législateur lui-même qui pourrait l'informer de ses intentions originaires. Les arguments de Michel Troper ciblent ce genre d'idéologie et la méthode qui la soutient, dont certaines formes sont encore vivantes.

Pour Dworkin, les cibles à critiquer sont celles des «originalistes» comme par exemple Robert H. Bork ou Anton Scalia. Ce courant crée aussi un rapport conceptuel entre l'interprétation «génétique» et la signification objective de la constitution: «The search is not for a subjective inten-

${ }^{11}$ En ce qui concerne le critique de cette distinction, ibid., pp. 190 et s. Cf. aussi la présentation de Troper, $D C$, pp. 263 et s.; $P h D$, pp. 104 et s. 
tion [...] When lawmakers use words, the law that results is what those words ordinarily mean» ${ }^{12}$.

Si l'on observe le niveau linguistique, il existe encore une «idéo-logique» simple, celle qui se rattache à la ratio legis. Selon les originalistes, l'intention originaire des pères fondateurs s'oriente vers certains buts et valeurs. Pour exprimer leurs préférences éthico-politiques, ils emploient les mots et les phrases dans leurs significations ordinaires. C'est la raison pour laquelle l'interprétation «génétique» est subsidiaire par rapport à l'interprétation «sémiotique». Cette dernière méthode se fonde sur l'hypothèse selon laquelle l'interprète peut simplement retrouver la «vraie» signification du texte depuis ses lettres. La méthode «génétique» est subsidiaire parce que selon les originalistes, si la méthode «sémiotique» ne conduit pas à un résultat adéquat, il est possible de retrouver les intentions originaires dans les documents de la «naissance» du message normatif. Au sein des deux méthodes, il existe un principe simple: même si le contenu sémantique du texte change, l'intention reste toujours la même ${ }^{13}$.

Les deux théoriciens élaborent donc des critiques «explicites» de l'idéologie conservatrice (légalisme français et «gouvernement of judiciary») ${ }^{14}$. Alors que Dworkin propose à sa place une autre idéologie, Troper constate simplement qu'il existe une divergence entre la réalité juridique et la vision qui est suggérée par cette méthode. Le désaccord entre Troper et les auteurs anglo-américains comme Dworkin dépasse les frontières culturelles juridiques. Tous deux admettent que la méthode «génétique» est une véritable méthode d'interprétation. Les juges interprètent le texte juridique même s'ils prétendent qu'ils ne sont que les «bouches de la loi». La seule question qui se pose est celle de savoir si la méthode «génétique» est une méthode plus «vraisemblable» qu'une autre méthode. Mais afin de prendre le «droit» —entendu «droits individuels» dans le cas de Dworkin ou la «science du droit» dans le cas de Troper- au «sérieux» les deux juristes considèrent également comme «sérieuse» cette idéologie conservatrice très forte fondée sur la «méthode génétique». Au sein du débat, les chemins méthodologiques divergent de nouveau.

3. Avant d'analyser cette critique, nous nous concentrerons d'abord sur le désaccord élémentaire concernant la position du théoricien vis-à-vis l'objet de ses recherches.

${ }_{12}$ Robert H. Bork, The Tempting of America. The Political Seduction of the Law, New York-London, The Free Press, 1990, p. 144.

${ }_{13}$ Ibid., p. 145.

${ }_{14} \mathrm{Ou}$ «historicisme» comme Dworkin le nomme: LE, pp. 359 et s. 
Tandis que chez le juriste français, le théoricien en «expliquant» le droit n'occupe qu'un «point de vue externe modéré» par rapport au discours qu'il observe, pour Dworkin, le théoricien qui tente de «comprendre véritablement» le phénomène juridique s'engage dans le discours qu'il «décrit». Chez Dworkin, il s'agit donc d'un point de vue «interne» au sens fort ${ }^{15}$.

La première étape de la méthode de «l'explication» de l'objet consiste à stipuler une définition et puis établir un modèle sur l'objet qui est hermétiquement séparé ${ }^{16}$. Troper, qui suit ce chemin, élabore donc une définition «stipulative» du droit sans penser qu'elle exprime l'essence du phénomène juridique. Elle n'est qu'un outil pour l'expliquer. Par contre, la finalité la plus importante de la «compréhension» consiste en une tentative de trouver l'essence de l'objet à étudier. Dworkin, quant à lui, ne se limite pas à cette explication. Il cherche plus exactement une notion du droit qui permet de comprendre l'essence de l'entreprise juridique ${ }^{17}$.

Or dans les deux cas, la question est de savoir si le droit produit dans un discours pragmatique-prescriptif n'est pas remis en cause. La spécificité de ce discours est que le but de tous ceux qui y participent n'est pas de «dire» (ou constater) mais de «parler» (ou faire).

Il existe trois types de discours pragmatiques qui semblent avoir une structure identique à celle du discours juridique: le discours quotidienne $(\mathrm{A})$, littéraire $(\mathrm{B})$ et scientifique $(\mathrm{C})^{18}$. La distinction entre eux permet aussi de relever certaines spécificités de la méthode «génétique» qui n’apparaissent dans leur pleine forme que dans le discours juridique, objet de la recherche du théoricien.

\section{A. La conversation quotidienne}

Bien que la conversation familiaire ou quotidienne soit intentionnelle, il n'est pas nécessaire de suivre la méthode «génétique», car le contexte pragmatique du discours ou la connaissance culturelle tacite donne des informations suffisantes pour comprendre les autres. Dans ce discours, le message arrive au récepteur sans médiation d'un texte et l'émission du

${ }^{15}$ Ibid., p. 55: «a social scientist must participate in a social practice if he hopes to understand it». Cf. aussi les pp. 14, 49, 68 ou p. 409: «philosophers are part of law's story».

${ }_{16}$ P.e., $P h D$, pp. 27 et $\mathrm{s}$.

${ }^{17}$ Il me semble que Dworkin pourrait être d'acord avec telle présentation. Cf. par exemple, $L E$, p. 413.

${ }^{18}$ Les trois formes du discours apparaît dans le deuxième chapitre de $L E$. 
message et l'acte de réception se passent presque simultanément et. Il est également facile d'identifier celui qui produit le message, car la conversation est faite entre des individus.

Par contre, dans le discours du droit, l'émission et la réception sont éloignées chronologiquement l'une de l'autre et la transmission du message normatif a lieu par l'intermédiaire d'un texte. Alors que nous trouvons dans le discours journalier des sujets empiriques parlants, dans le discours juridique ils sont l'auteur et le lecteur, autrement dit, le plus souvent, le législateur (qui ne se réduit pas à une personne physique, ce qui le rend difficile à «identifier») et le juge qui entrent en conversation.

Alors que la conversation familière n'a pas de dimension temporelle marquante, la méthode «génétique» de ce point de vue rappelle les deux faces de Janus. Elle recommande que celui qui interprète prenne en considération, d'une part, la reconstruction diachronique de l'intention de l'auteur et, de d'autre part, la construction synchronique de la signification d'un texte. Le processus interprétatif semble être tout à fait cognitif: après avoir identifié l'auteur et constaté son intention, il ne reste au lecteur que de l'appliquer.

\section{B. La conversation littéraire}

Le processus «cognitif», la similitude entre la position de celui qui produit le message (qu'il soit l'auteur et le législateur) et de celui qui l'interprète (qu'il soit le lecteur et le juge), et la similarité de l'objet de l'interprétation (l'œuvre d'art ou les textes juridiques) conduisent les philosophes du droit adhérant au mouvement Law and Literature à unifier la théorie de l'interprétation littéraire et juridique ${ }^{19}$. Sous leur influence Dworkin dit: «judges [...] are authors, as well as critics» ${ }^{20}$. En unifiant ces deux discours avec le discours théorique sur le droit, il soutient que les propositions juridiques, les propositions sur le droit et les jugements esthétiques sur une œuvre d'art —en tant que discours pragmatique— sont susceptibles d'être vraies ou fausses.

Une proposition sur le droit est «vraie» si elle correspond à une pratique sociale particulière ${ }^{21}$, donc au «bon» raisonnement des participants à

19 En générale cf. Ronald Dworkin, A Matter of Principle, Cambridge MA, Harvard University Press, 1985, Ch. 6. Pour le critique cf. FisH, note 9, pp. 87-149.

${ }^{20} L E$, p. 229.

${ }^{21}$ Ou même avec «les principes de la justice et de l'équité». Cf. ibid., p. 225. 
travers lequel ils justifient leur comportement. Malgré ses critiques contre les «originalistes», Dworkin estime que l'intention —par conséquent la méthode «génétique»- reste une notion tout à fait centrale ${ }^{22}$. Sur ce point, l'analogie entre le droit et la littérature, n'est pas suffisamment justifiée pour Michel Troper ${ }^{23}$, et ceci pour plusieurs raisons. Premièrement le droit hiérarchise l'interprétation juridique selon l'auteur alors que les lecteurs sont égaux dans le champ de la littérature; deuxièmement, une décision est produite après l'acte d'interprétation dans le champ normatif, car il existe une obligation juridique de décider (ce qui n'est pas vraie dans le cas de l'interprétation d'un livre ou d'un poème); et enfin, les romans ou poèmes ne disposent pas de l'autorité qui est attribuée au Code et aux lois par le système juridique (en littérature, il est bien évident que personne ne possède le pouvoir d'attribuer une telle autorité à une œuvre d'art).

En ce qui concerne donc le discours familier, l'acte d'interprétation au sens stricte n'a pas lieu parce que, à proprement parler, il n'y pas de faits (un texte) à interpréter. Dans le cas du discours littéraire, la situation est plus complexe puisqu il est similaire au discours juridique dans la mesure où l'observateur doit déterminer son but exact. Il s'agit d'un choix entre «compréhension» et «explication». Celui qui tente de comprendre une œuvre littéraire ou juridique cherche véritablement son essence. Pour cette recherche, il faut englober les analyses et les intentions des agents du discours. Celui qui ne cible que l'explication du discours ne suppose pas qu'il existe une essence du discours dont l'intention est exprimée. Son analyse donc ne dépasse pas une analyse sémantique du discours. Ainsi tandis qu' «expliquer» une règle du Code civil ou un passage de la Bible ou du Catch-22 consiste à présenter les significations possibles du texte, «comprendre» le Code civil, la Bible ou le Catch-22, c'est d'abord déterminer les intentions de l'auteur et puis chercher l'essence de l'œuvre. Au sein des deux méthodes se trouvent deux théories linguistiques différentes.

Dworkin et les «intentionnalistes», suivant directement ou indirectement John L. Austin, justifient la détermination du droit par l'importance des actes performatifs, donc par les actes de la langue intentionnelle qui possèdent une force illocutoire. Si l'observateur ou le participant (ou encore, l'observateur-participant) tente de comprendre ce qu'un autre agent dit dans un discours pragmatique, il semble nécessaire également de cher-

\footnotetext{
${ }^{22}$ Ibid., pp. 55 et s.

23 «Une théorie réaliste de l'interprétation» et «Les juges pris aux sérieux. La théorie du droit selon Dworkin» dans TDDE, pp. 70 et 47.
} 
cher ses intentions. Dans la ligne de Dworkin, certains autres théoriciens du droit vont jusqu'à dire qu'il est possible de découvrir une contradiction logique si l'intention du sujet-parlant du discours pragmatique est contraire à ce qu'il dit. Ils recommandent que le travail du théoricien soit de rendre explicite de telles contradictions ${ }^{24}$.

Par contre, les «antiintentionnalistes» maintiennent la distinction saussurienne entre «la langue» et «la parole», et souscrivent à la spécificité de la langue dans son caractère autosuffisant. En appliquant une telle démarche linguistique dans un autre domaine - par exemple en science du droit- le savant ne dépasse pas l'analyse sémantique du discours vers la pragmatique comme les «intentionnalistes» le font. Ainsi, la détermination de l'intention est impossible ou bien elle pourrait être possible en dehors de l'analyse scientifique.

La démarche de Troper devrait être classifiée clairement comme «antiintentionnaliste»: «la théorie réaliste n'admet [pas] [...] la possibilité de connaître autre chose que l'expression de ces normes dans le langage» ${ }^{25}$. L'explication dans la science porte sur les faits, et les faits sont des significations des textes juridiques déterminés par les autorités, qui seules produisent des effets dans le système juridique. Le théoricien ne cherche donc pas les intentions qui conduisent à naissance d'une décision - la genèse de la norme au sens habituelle-, mais il indique les causes qui déterminent la situation dans laquelle une décision est prise.

\section{La conversation scientifique}

Ce troisième type de discours consiste à affirmer que «l'intention» ne joue aucun rôle. Le discours scientifique est par nature nullement pragmati$q^{2} e^{26}$. On retrouve ce type de démonstration dans d'autres raisonnements. Ainsi Dworkin soutient que «[S]cientific interpretation is not really an interpretation at all $\rangle^{27}$. Evidement, un positiviste pense exactement le contraire.

Dworkin va même encore plus loin. La raison pour laquelle il rejette la méthode «génétique» est qu'elle n'établit pas forcément la meilleure

${ }^{24}$ Sur la notion de la «contradiction performative» cf. p.e. Robert ALEXY, «On Necessary Relations Between Law and Morality», II Ratio Juris (1989) 2, pp. 167-183 et s.

25 «Réplique à Otto Pfersmann» dans Analisi e diritto. Ricerche di giurisprudenza analitica sous la dir. P. Comanducci et R. Guastini, Torino, Giappichelli, 2002-2003, p. 300.

${ }^{26} \mathrm{LE}, \mathrm{pp} .86$. et s.

27 Ibid., p. 51. 
forme de raisonnement du discours pragmatique juridique. La référence «historique» ne donne pas une réponse plausible en cas d'un «désaccord théorétique» (compris comme un désaccord sur la nature ou la définition du droit). Pourtant, les «auteurs» (législateur ou les «pères fondateurs») occupent du point de vue chronologique les premières places du «roman à la chaine» juridique ayant une liberté sans limite afin de créer le droit ${ }^{28}$. L'interprétation, cherchant à promouvoir la «meilleure forme», prend en considération de façon égale l'intention des différents auteurs (ou «l'histoire législative»). Mais, contrairement aux «originalistes», Dworkin soutient qu'il est nécessaire d'englober la recherche de l'interprète à d'autres facteurs comme celui de la «cohérence» du roman de la chaîne aux principes «de justice et d'équité».

Dans l'approche troperienne, le théoricien ne devrait pas tenter de «comprendre véritablement» le droit. En ce sens, il pense qu'il n'y a pas une essence du droit qui permette de le comprendre. De même, il ne s'intéresse pas non plus aux actions et aux motivations des participants au discours pragmatique. L'objet de la théorie du droit à ses yeux est d'indiquer les critères du discours scientifique sur le droit. Dans le cadre de ce discours, le théoricien a pour fonction de justifier ou d'écarter l'existence des faits produits par les actes de la volonté afin d'élaborer un modèle scientifique ${ }^{29}$.

4. Bien qu'il soit possible de préciser l'une ou l'autre méthode «classique» de l'interprétation sans faire référence à l'intention, il n'est pas possible d'élaborer une théorie «génétique» de l'interprétation sans supposer l'existence d'une signification objective du texte juridique. Par rapport aux autres théories de l'interprétation, la spécificité de la méthode «génétique» réside dans le fait que la reconstruction de l'intention de la volonté du législateur, exprimée dans un discours pragmatique, indique le sens objectif du texte.

Dans le cas de la méthode «génétique» de l'interprétation, on trouve des précisions différentes. Parmi elles, nous en choisirons trois. Nommons-les «personnificatrice», «mentaliste» et «formaliste». Chacune des trois versions correspond à une méthode de recherche de l'intention: la première cherche ce que le législateur «voulait dire», la deuxième tend à découvrir ce que le législateur «pensait vouloir dire», et le but de la troi-

\footnotetext{
${ }^{28}$ Ibid., pp. 228 et s.

29 «Une théorie réaliste de l'interprétation» dans TDDE, p. 70: «[L]'interprétation est une activité qui consiste soit a indiquer, soit a déterminer la signification d'une chose».
} 
sième est de trouver ce qu'il «aurait voulu dire». Vu que les deux premières versions supposent la possibilité d'identifier un auteur «factuel», elles forment une seule classe, contrairement à la troisième, qui est ouvertement «contrefactuelle» et qui est identique à la deuxième classe.

\section{A. Les versions «factuelles»}

Selon la version «personnificatrice», il est possible et nécessaire d'identifier le législateur et son intention unique. Selon la version «mentaliste», même si le législateur ou l'auteur du texte normatif est introuvable, il est possible et nécessaire d'identifier son «état mental».

Les deux premières versions -inspirées très probablement de la psychologie individuelle- ${ }^{30}$ conçoivent le législateur comme une personne réelle ayant une intention unique. Pour trouver son intention univoque l'outil classique pour l'interprète est la consultation du législateur «réel» (référé législatif) ou —s'il est «mort»- des documents d'archives des débats parlementaires. Dans une controverse, Troper souligne que cette méthodologie est encore vivante aujourd'hui: «L'interprétation génétique repose sur une connaissance de la volonté réelle de l'auteur du texte. On suppose que cette volonté a été mise à mal dans le texte même, ambigu ou vague et qu'on peut le connaître à travers les travaux préparatoires. [Même si les juristes] y sont hostiles, surtout lorsque la loi à interpréter est ancienne et que le législateur historique n'a guère pu exprimer sa volonté sur une question qu'il ignorait [...] [Néanmoins] les arguments tirés de travaux préparatoires ne sont en général nullement absents du débat» ${ }^{31}$. Selon Troper, la version «personnificatrice» comme la version «mentaliste» sont toutes deux faciles à critiquer: ou bien on admet qu'il est impossible d'identifier l'auteur intellectuel ou les véritables auteurs du texte, et alors la question de l'intention perd son intérêt, ou bien cette identification est possible, mais étant donné la multiplicité des sujets physiques représentant «l'auteur», la détermination de l'intention unique de l'auteur est exclue ${ }^{32}$.

Deux exemples peuvent attester de cette difficulté: le cas de l'adoption d'une loi lorsque le texte est préparé par l'administration et adopté par les membres du parlement pour des raisons différentes, ou celui du référen-

${ }^{30}$ Cf. Lon L. Fuller, The Morality of Law, New Haven-London, Yale University Press [rev. ed.], 1969 , pp. 81 et s.

31 «La signature des ordonnances: fonctions d'une controverse» dans PTJE, p. 278.

32 «Une théorie réaliste de l'interprétation» dans TDDE, p. 73. 
dum où l'on consulte des millions d'électeurs (qui correspondent donc à l' auteur du texte). Dans les deux cas, il est impossible de parler d' «état mental», et par conséquent de déterminer une même et unique intention ${ }^{33}$. Quant à Dworkin, il déclare simplement que la vieille idée de «l'état mental» de l'auteur (ou du législateur) ne joue aucun rôle décisif dans l'interprétation d'un texte juridique ${ }^{34}$.

\section{B. La version «contrefactuelle»}

Cette forme suppose qu'il est possible d'identifier la «véritable signification» d'un texte en supposant contrefactuellement l'existence de l'intention de sorte que deviennent identiques la formule exprimant la classe générale ou l'extension de l'intention supposée et la classe construite.

Dans ce cas même, les éléments «plausibles» de cette méthode ne sont plus relatifs à l'application, mais relève bien d'une véritable théorie de l'interprétation ${ }^{35}$. Evidemment, selon Dworkin, cette version n'est guère plus plausible. Les travaux de ce dernier visent à élaborer non seulement une théorie générale et formelle du droit, mais aussi une philosophie —ou comme il la nomme une «théorie normative»— ${ }^{36}$ qui incorpore une théorie morale «académique» ${ }^{37}$.

Dworkin ne cherche pas l'auteur empirique — malgré le fait qu'il renvoie parfois aux auteurs de la constitution ou de ses amendements- mais il le conçoit comme une construction théorique. L'auteur «construit» représente donc les individus ensemble, tous ceux qui participent dans une «pratique sociale». Cette pratique sociale est véritablement un discours pragmatique et ses caractères argumentatifs et interprétatifs marquent son caractère spécifique. Chaque participant exprime ses intentions dans une forme de raisonnement et en même temps il donne une signification - ayant une attitude interprétative- à son propre comportement. Dans cette conversation, l'intention donne une structure formelle à toutes les exigences interprétatives des agents. Une telle conversation n'est possible que si les membres de la communauté partagent «une certaine

33 Ibid., pp. 72 et s.

${ }^{34}$ LE, pp. 57 et 229.

35 «Une théorie réaliste de l'interprétation» dans TDDE, p. 73.

36 TRS = Ronald DworkIn, Taking Rights Seriously, Cambridge-Massachusetts, Harvard University Press, 1977, pp. vii et s.

${ }^{37}$ Ibid., pp. 285 et s. 
conception de la vie» afin de comprendre les arguments des autres en cas de désaccord ${ }^{38}$.

Sur cette base, il est possible de construire une notion abstraite de «l'auteur» qui, selon Dworkin, est la communauté personnifiée. Le droit est conçu comme intégrité «narrative» —respectée par les auteurs «politiques» différents (par les «framers of the constitution» et par les juges)—, parce que la communauté personnifiée est un agent moral qui possède une responsabilité collective et abstraite à l'égard de certains principes, comme celui de l'obligation d'impartialité des agents de l'État ${ }^{39}$. L'intention devient particulièrement abstraite, lorsqu'on la définit comme étant celle de la communauté personnifiée, comme dérivée des intentions de chaque individu de vouloir vivre ensemble dans une «communauté fraternelle». Malgré tout cela, elle est susceptible d'être identifiée par le théoricien.

«L'auteur» chez Dworkin, mais aussi chez Troper est une construction desontologisée ${ }^{40}$, c'est-à-dire qu'il n'est pas susceptible d'être identifié empiriquement comme dans le cas de la conversation «quotidienne». Chez Dworkin, il est remplacé par les principes de la philosophie morale ou politique, chez Troper, par le droit lui-même: l'auteur n'est plus qu'une construction juridique, qu'une «fiction».

L'exemple de l'auteur de la constitution peut être éclairant. Dans son manuel du droit constitutionnel, Troper le définit comme «pouvoir constituant ${ }^{41}$. Il s'agit d'une notion juridique liée à la distinction entre deux types de constitutions: «rigide» et «souple». Ni les «principes» moraux, ni la détermination sociologique ou politique ne comptent dans la détermination théorique de «l'auteur» de la constitution — le «peuple», la «nation»- car selon la position constante du positivisme juridique auquel Troper lui-même adhère explicitement, c'est la constitution elle-même qui crée les «principes» et les notions tels que le «peuple» ou la «nation» ${ }^{42}$. (Rien n'empêche évidemment l'analyse de l'histoire constitutionnelle ou de la sociologie politique dont l'objet est d'identifier ceux qui étaient les sujets psychiques qui ont écrit et voté le texte de la constitution, ou même ceux qui étaient représentés par les auteurs intellectuels).

38 Ibid., p. 58

39 Ibid., pp. 167 et s.

${ }^{40}$ Les deux types de desontologisation se trouvent chez Antonio NegRI, Le pouvoir constituant. Essai sur les alternatives de la modernité, trad. de l'italien par E. BALIBAR et F. Matheron, Paris, Presses Universitaires de France, 1997.

${ }^{41} D C$, pp. 42 et s.

${ }^{42}$ Cf. «La titulaire de la souveraineté» dans TDDE, pp. 283 et s. 
Le contenu matériel des notions est donc tout à fait contingent. La théorie du droit, formelle et générale, ne pourrait que montrer ce qui existe, ce n'est qu'un type de contrainte conceptuelle. Ainsi, le théoricien peut montrer que les textes constitutionnels successifs ne pourraient utiliser d'autres concepts que ceux qu'ils avaient déjà inventés (tels que le «peuple» ou la «nation»).

Pourtant, le juge construit dans sa meilleure forme une classe de choses qui correspond raisonnablement à l'intention du législateur, donc à ce qu'il «aurait voulu dire». Il prétend subsumer les faits sous une classe imaginaire. Cette version est ouvertement une théorie de l'interprétation et il ne s'agit plus de se référer à la théorie qui soutient que les juges n'interprètent pas, mais appliquent la loi. Cependant, le théoricien est confronté à un problème similaire: si l'intention hypothétique prouve que le juge est en face de faits qui n'étaient pas prévus au moment de la naissance de la norme, le juge commettrait un déni de justice en refusant de trancher le litige - ce qui est défendu, au moins dans le système juridique français_ ${ }^{43}$ lorsque la classe et les faits, ne correspondent pas. Ce genre de situation n'est pas extraordinaire: presque toutes les affaires qui concernent les dommages causés par une nouvelle technique ou machine tombent dans cette situation ${ }^{44}$.

Mais supposons, qu'il soit possible de comprendre les faits sous la catégorie. Dans ce cas, le juge établira un syllogisme juridique où dans la prémisse majeure est établie (contrefactuellement) suivant l'intention hypothétique du législateur, et les faits du cas donné constitueraient la prémisse mineure. Pour attester cette hypothèse, certains théoriciens montrent que le juge décide véritablement de telle façon, autrement dit, il décide en établissant une inférence logique entre les prémisses et la conclusion. La théorie «réaliste» (ou positiviste) rejette cette analyse. Plusieurs arguments possibles peuvent expliquer le rejet —ainsi par exemple le fait de raisonner même contre la possibilité d'une logique juridique $-{ }^{45}$, mais dans un souci de brièveté, nous ne retiendrons que l'argument décisif.

Pour les réalistes, justifier empiriquement que l'intention du législateur s'orientait, non seulement, vers l'établissement d'une classe générale (la prémisse majeure), mais aussi vers chaque cas particulier (la prémisse mineure) est clairement impossible. Et même si cela était possible, il fau-

\footnotetext{
${ }^{43}$ Notamment en vertu de l'article 4 du Code Civil.

44 «Une théorie réaliste de l'interprétation» dans TDDE, p. 73.

${ }^{45} \mathrm{PhD}$, pp. 112 et s.
} 
drait justifier que le juge établit une déduction logique entre deux actes de volonté. Cette solution est exclue parce que les normes ne sont pas des vérités, mais des commandements.

Pour résoudre le problème, deux chemins possibles semblent envisageables. Le premier est l'interprétation constructiviste dworkinienne qui met en jeu l'intention de la «communauté personnifiée» parmi d'autres facteurs. Cette interprétation apparaît dans le discours pragmatique. Sur le plan épistémologique, elle admet que la théorie perd son caractère scientifique. L'autre chemin consiste à abandonner totalement la tâche traditionnelle de la science du droit comme le préconise Troper et de ne plus chercher les méthodes d'interprétation ni la possibilité d'une logique juridique. Elle consiste à se focaliser sur les faits susceptibles d'être falsifiés ou justifiés. Cette approche, qui semblait dangereuse du point de vue de la démocratie, oriente la théorie du droit vers une nouvelle méthode de travail du théoricien. Pourtant, elle demeure en même temps tout à fait classique, si on la compare avec la tradition du positivisme juridique.

5. Il est intéressant de constater que les trois types de critiques se fondent sur une séparation rigoureuse entre le discours en droit («le droit») et le discours sur le droit («la science du droit»). Cette distinction est faite à l'égard des effets de l'interprétation. Tandis que dans le premier discours, les actes d'interprétation produisent des effets juridiques sans exception, le droit n'attribue aucun effet pour le deuxième. Cette distinction classique, qui se trouve chez Kelsen, s'harmonise avec la classification «binaire» entre les «modes» d'interprétation «authentique» et «scientifique» ${ }^{46}$.

En ce qui concerne la signification du mode «authentique» de l'interprétation, des positivistes depuis Kelsen se sont éloignés du sens originaire de cette notion. A l'origine la mode de l'interprétation authentique renvoyait à celle qui était produite par «l'auteur» (législateur) de la norme. Cette méthode, dans ce sens, était complémentaire de la méthode «génétique» ${ }^{47}$. Or, pour Troper et pour les autres positivistes ${ }^{48}$, la méthode

46 Théorie pure $d u$ droit, trad. allemand par H. ThévenAz, Paris, Neuchatel, 1988, pp. 152 et s.

47 En ce sens cf. par exemple Thomas Marie JoSEPH GousSET, Théologie morale à l'usage des curés et des confesseurs. Traité des actes bumains, de la conscience, des lois, des péchés, des vertus et du décalogue, t. I, Paris, J. Lecoffre, 1848, pp. 68-69 et 178. Cf. également Ost et Kerchove, note 2, pp. 170, 179 et s.

${ }^{48}$ Comme Ferrajoli «qui distingue entre l'interprétation doctrinale qui est faite par le juriste "spectateur" et contemplant la signification du langage juridique, interprétation "opérative", qui est faite par du juriste "acteur" participant à sa création». Cité par OsT et KERCHOVE, ibid., p. 171. 
«authentique» de l'interprétation est celle qui produit des effets juridiques en émanant d'une autorité dont la décision n'est pas susceptible d'être contestée. La classification binaire des méthodes d'interprétation se fonde sur la séparation rigoureuse des deux discours: le discours (descriptif) des théoriciens et le discours pratique (prescriptif) des juristes.

En ce qui concerne le premier discours, l'objet de l'interprétation n'est pas la normecomme le suggère Kelsen, mais un énoncé ou le texte luimême: «[L]a volonté du législateur ne produit que des énoncés, alors que la norme exprime la volonté de l'interprète» ${ }^{49}$.

La critique de la méthode «génétique» de l'interprétation se développe aussi autour d'une thèse selon laquelle le texte même ne porte pas une seule signification objective à découvrir, mais tout au contraire, comporte des significations illimitées du texte déterminées par l'autorité. La norme elle-même n'est rien d'autre qu' «une signification d'un énoncé» ${ }^{50}$ ou «la signification prescriptive du texte» ${ }^{51}$.

La thèse choquante, celle qui précise qu' «avant l'interprétation, il n'y a pas de norme, mais un simple texte» ${ }^{52}$ exprime parfaitement le rejet général de la soi-disant théorie de l'interprétation. A défaut de processus privilégié permettant de trouver la «bonne» signification du texte, il ne peut exister de méthode privilégiée de l'interprétation. La diversité des méthodes d'interprétation justifie elle-même la liberté de l'interprète.

Des conséquences sérieuses de cette thèse peuvent être tirées —d'ailleurs critiquées vivement et à plusieurs reprises par Dworkin-. Ceci signifie que chaque décision juridique est rétroactive ${ }^{53}$, et il est impossible de parler de la période «préinterprétative» ${ }^{54}$ de l'interprétation juridique où la volonté et l'intention du législateur pourraient être prises en considération. En générale, ce que le professeur américain propose, est une démarche «protestante» dans le domaine de l'interprétation juridique. Ceci pourrait également signifier que l'interprétation «authentique» (au sens positiviste de Kelsen et Troper) détermine le débat juridique en exprimant le «dernier mot», mais il n'est pas forcement le meilleur «mot» ${ }^{55}$.

\footnotetext{
${ }^{49} \mathrm{PhD}$, p. 86 et ch. IV

50 Ibid., p. 76.

51 Ibid., p. 105.

52 Ibid., p. 106.

53 TRS, ch. I.

54 LE, pp. 65 et s.

55 Ibid., p. 413.
} 
6. La description scientifique démasque l'idéologie «illusionniste»: même si les juges pensent qu'ils ne sont que les «bouches de la loi», ils sont véritablement libres vis-à-vis de l'intention supposée du législateur ou du texte; plus précisément en face des significations à donner. Cette «liberté» au sens juridique ne signifie rien d'autre que le pouvoir discrétionnaire, «c'est-à-dire le droit d'un sujet ou d'un organe d'adopter la conduite qu'il veut ou de donner le contenu de son choix aux normes qu'il a le pouvoir d'énoncer» ${ }^{56}$.

En somme, le texte ne peut pas limiter le pouvoir de celui qui l'interprète. Les positivistes — comme l'évêque Hoadly, Kelsen (pendant sa période tardive) et Michel Troper — ${ }^{57}$ admettent donc que «le véritable auteur n'est pas l'auteur du texte, c'est l'interprète» ${ }^{58}$. Ainsi, dans le cas d'une constitution, les auteurs ne sont ni les «pères fondateurs», ni «le peuple» qui adopte le texte par référendum, mais bien les pouvoirs publics, en l'occurrence le Président de la République, le Parlement et le Conseil constitutionnel ${ }^{59}$ qui peuvent donner à ce texte «n'importe quelle signification» ${ }^{60}$. Pourtant une question demeure: est-ce que le positiviste doit également admettre que le juge est un tyran qui agit librement selon ses caprices?

Le modèle scientifique de Michel Troper montre que la décision juridique, dans un système juridique bien structuré, ne laisse pas de place pour l'abus de pouvoir car il existe des «contraintes multiples» existant est indépendamment de l'intention des auteurs des textes juridiques.

Il distingue entre deux types de contraintes. Au sens fort, il s'agit de règles constitutives fondées sur le rapport entre le but et le moyen. Si un individu veut accomplir un certain but - par exemple un contrat valide ou changer son état civil par un acte de mariage- il est s contraint de poursuivre certaines règles imposées par un système normatif. Il s'agit de contraintes parce que s'il veut réussir, l'individu est forcé de ne pas choisir d'autres moyens, donc de ne choisir que le moyen ordonné. Bref, les règles

56 «Une théorie réaliste de l'interprétation» dans TDDE, p. 83.

57 Hans Kelsen, General Theory of Law and the State, New York, Russel \& Russel [1945], 1961, p. 153. Cf. aussi BorK (note 22), qui cite les autre théoriciens américains, pp. 176 et 390.

58 «Justice constitutionnelle et democratie» dans PTJE, p. 334. Cf. aussi LuHMANN, «Les événements pertinents, dans le cas du droit, ce sont les décisions», cité par Pierre GuIBENTIF, «Niklas Luhmann Observateur du droit», cité dans Niklas Lubmann Observateur du droit sous la dir. André-Jean Arnaud et Pierre Guibentif, Paris, Libraire Générale de Droit, 1993 , p. 41 [Collection Droit et Société].

59 Ibid., p. 332.

${ }^{60} \mathrm{PhD}, \mathrm{p} .111$. 
constitutives sont celles «qui ne se borneront pas à prescrire une conduite, mais la constituent en ce sens que c'est l'observation de ces règles, qui permettent de la qualifier» ${ }^{61}$.

Certes, du point de vue «antiintentionnaliste», l'existence de ce type de contraintes qui fonde la doctrine des actes performatifs n'est pas recevable pour deux raisons. D'un côté parce qu'en l'admettant, il faudrait retourner chercher et déterminer les intentions des agents au niveau pragmatique, thèse qui a déjà été rejetée. D'un autre côté, parce que le système juridique lui-même n'opère pas selon ce modèle intentionnaliste. Une décision juridique quelconque restera valide jusqu'à ce qu'elle soit invalidée par une autorité compétente supérieure.

Outre une contrainte au sens fort, il demeure un mécanisme de contrainte au sens faible. Il est «faible», parce qu'il est possible d'agir contrairement à lui: un Parlement dans une société démocratique pourrait adopter une loi ouvertement raciste afin d'utiliser la loi comme un moyen de servir certains buts de la majorité. Il ne le fait pas parce que la Cour constitutionnelle pourrait être saisie par le président de la République si le système connait le contrôle a priori, ou bien par des individus ayant un intérêt à agir, dans les systèmes où le contrôle a posteriori existe, les. Cette contrainte existe aussi à l'égard de la Cour: si elle interprétait la Constitution de manière excessive- selon les caprices des juges agissant collectivement comme un monarque absolu - elle perdrait vite sa légitimité. Le Parlement, agissant comme «pouvoir constituant», pourrait alors remettre en question l'utilité et l'existence même de la Cour constitutionnelle ${ }^{62}$.

Un autre mécanisme de contrainte au sens faible est établi par la jurisprudence. La chaîne des actes d'interprétation «authentique» établie aussi une contrainte, notamment en vertu de la nécessité de cohérence ${ }^{63}$. Les juges - comme les législateurs- sont factuellement sous la contrainte de motiver leur décision en adoptant les directives, les concepts et les modes de raisonnements établis par les juridictions suprêmes. Ces dernières sont liées également par leur jurisprudence car la cohérence aide à soutenir leur propre légitimation dans le système politique. Il arrive très souvent que les autorités suprêmes contrôlent les autres interprètes dans l'application de certains modes d'interprétation, comme par exemple celui qui fait référence à l'intention du législateur. Néanmoins, la cohé-

\footnotetext{
${ }^{61}$ «La liberté d'interprétation du juge constitutionnel» dans TDDE, p. 95.

${ }^{62}$ Ibid., p. 110.

${ }^{63}$ Ibid., pp. 111 et s.
} 
rence (ou la «raison juridique») parfois cristallisée dans cette jurisprudence, ne pourrait pas non plus invalider elle-même la décision, parce qu'en droit: autoritas (l'autorité ayant la compétence), non veritas (le «raisonnement plausible») facit jus.

La «cohérence» se situe aucœur de la théorie de l'interprétation de Dworkin et des autres auteurs «post-positivistes». Selon sa fameuse image, les juges agissent à travers la jurisprudence comme les co-auteurs de la norme, occupant des places chronologiquement d'un roman à la chaîne. Dans cette entreprise commune, le législateur (ou le pouvoir constituant) occupe la première place ayant liberté pleine d'inventer le début de l'histoire, donc le texte de la norme ou de la constitution. C'est lui qui établit les éléments constitutifs de «l'histoire juridique» qui «termine» avec «la seule bonne réponse» à la question juridique à résoudre. L'intention —ou plus généralement, «l'histoire législative» d'une règle juridique- devrait être prise en considération par ceux qui interprètent, mais pas de manière exclusive. Il faut que l'interprète la complète avec les principes cristallisés dans la pratique juridique et avec la cohérence présente dans les cas précédents. Mais dans ce cas aussi, la décision juridique n'est pas valide parce qu'elle est élaborée suivant les lois de la logique, mais bien parce qu'elle a été établie par une autorité ayant la compétence de l'interpréter.

Chez Dworkin, la décision juridique n'est pas une véritable décision, mais la conclusion d'un raisonnement juridique dérivée par l'autorité compétente. La notion de pouvoir ne joue aucune rôle dans sa théorie de l'interprétation, elle est remplacée par la raison. Par contre, pour Troper, le terme «pouvoir» est entendu au sens négatif. Il ne s'agit que d'une autolimitation justifiée simplement par la raison pratique ou l'efficacité: la modération du Roi ou du président de la République face à l'adoption d'une loi inacceptable ou —dans le cas du président- de ne pas trop souvent estimer les circonstances comme constituant une «menace grave et immédiate». Leur autolimitation est raisonnable et effective parce que le Roi est en faveur du principe selon lequel «une loi valide fondée sur un compromis, est préférable», et parce que le président de la République voudrait éviter par exemple un procès fondé sur l'accusation d'abus de pouvoir. Cependant, ce mécanisme fonctionne surtout dans le champ extra-juridique puisqu'il est possible — comme dans le cas du roi de Xvirème siècle en France- qu'il n'y ait pas de sanction directe et juridique contre la transgression du pouvoir. Dans ce même sens, il était également possible de chercher les motivations ou les intentions des interprètes ou de relever le caractère moral du Roi ou du président de la République. 
La science du droit entend le terme «pouvoir» au sens positif. Dans ce sens, le pouvoir consiste à déterminer le comportement des autres qui ont également le pouvoir d'interpréter. Nous avons déjà mentionné les exemples du président de la République française, du Parlement et de la Cour Constitutionnelle, interprètes qui se limitent les uns les autres. Il ne s'agit donc pas du cas dans lequel les agents agissent sous la contrainte pour éviter les conséquences indésirables, mais ils agissent plutôt en prenant en considération la prévisibilité et la stabilité du système juridique où les agents connaissent les limites de leur pouvoir discrétionnaire et les individus ont la chance de pouvoir prévoir les conséquences légales de leur comportement. Ce sont des contraintes factuelles, parce que les décisions sont fondées sur l'acte de volonté de l'autorité. Toutefois, sur la base de la volonté, on trouve les méthodes et les concepts juridiques — donc les «contraintes juridiques»— qui déterminent le comportement des interprètes. La contrainte vient du fait que l'interprétation a un caractère collectif qui est établi par les rapports de force.

7. Afin de soutenir la séparation entre le discours prescriptif du droit et le discours descriptif sur le droit, le théoricien qui occupe un point de vue «externe» par rapport au discours juridique n'intervient pas par ses recommandations dans le travail de ceux qui possèdent le pouvoir d'interpéter le droit. Ainsi, il est exclu de qualifier cette méthode «positiviste» puisque aucune méthode positiviste de l'interprétation juridique n'existe. Le théoricien ne doit pas se focaliser sur les significations «adéquates» des textes juridiques, mais il faut qu'il indique la signification des normes —notamment celle déterminée par une interprétation «authentique»-. A partir de ces travaux, le théoricien positiviste élabore une description rigoureuse du mécanisme interne de la production du droit. Il ne s'intéresse nullement aux intentions ou aux effets sociaux ni au discours pragmatique juridique, ni à sa propre parole. Ainsi, une théorie «positiviste» se situe hors de la classification idéologique.

Mais est-ce qu'il y a une place pour les méthodes de l'interprétation, comme celle de la méthode «génétique»? Sans doute, il existe d'autres discours à côté du droit et de la science du droit. Le plus important parmi eux est celui de la dogmatique juridique. Ce discours est essentiellement un mélange de discours «en» et de «sur» le droit. Les modes d'interprétation - y compris la version «génétique»— appartiennent à ce domaine.

Ce domaine est le lieu de naissance des recommandations interprétatives, d'untype particulier de raisonnement et de directives. La dogmatique juridique n'est donc pas scientifique, «puisqu'elle se définit par la mise en oeuvre 
d'évaluations» ${ }^{64}$. Elle présuppose nécessairement des idées non-falsifiables ou même de nombreuses adhésions idéologiques comme par exemple la rationalité du législateur ${ }^{65}$. Contrairement aux indications scientifiques, les opérations interprétatives dogmatiques vont plus loin que la description des normes juridiques en vigueur. Elle les systématise et les interprète.

Entre la science et la dogmatique juridique, il y a une deuxième différence autre que dans l'engagement moral ou politique. Tandis que l'objet de la science juridique porte sur les normes juridiques en vigueur (plus précisément les significations «officiellement établies»), celui de la dogmatique juridique porte sur les normes juridiques applicables. La méthode du premier est la description, et du deuxième la recommandation ${ }^{66}$. Les opérations intellectuelles de la dogmatique juridique $-\mathrm{y}$ compris les règles de la méthode «génétique» de l'interprétation- parfois explicitement, parfois implicitement servent des buts idéologiques. Elles n'indiquent pas simplement le sens des normes juridiques, mais servent également à les déterminer. Elles ne présentent pas seulement les mécanismes internes de la production décisionnelle des actes du pouvoir, mais elles modifient aussi les mécanismes en les critiquant. C'est exactement le but du Profeseur américain qui est rejetté par le théoricien français.

En somme, la différence décisive entre Troper et Dworkin en ce qui concerne le fond du problème de la méthode génétique est que, pour le deuxième, la dogmatique juridique fait partie de la théorie du droit, alors que pour Troper, elle est séparée de la théorie du droit et cette séparation demeure une des garanties parmi les plus importantes en ce qui concerne la neutralité axiologique de la science du droit. De façon plus abstraite, on pourrait dire que l'horizon de l'interprétation de la dogmatique juridique chez Dworkin est remplacé par l'horizon de la décision de la science juridique chez Troper.

\footnotetext{
${ }^{64}$ «Science du droit et dogmatique juridique» dans TDDE, p. 13.

${ }_{6} \mathrm{PhD}, \mathrm{p} .60$.

${ }_{66}$ Ibid., p. 61.
} 